\title{
Prevalence of high-risk HPV genotypes, categorised by their quadrivalent and nine- valent HPV vaccination coverage, and the genotype association with high-grade lesions
}

María Paz-Zulueta* ${ }^{*}$, Ledicia Álvarez-Paredes², Juan Carlos Rodríguez Díaz³, Paula Parás-Bravo', Ma. Encarnación Andrada Becerra ${ }^{4}$, José María Rodríguez Ingelmo ${ }^{5}$, María Montserrat Ruiz García ${ }^{6}$, Joaquín Portilla ${ }^{7}$ and Miguel Santibañez ${ }^{1}$

\begin{abstract}
Background: The new nine-valent vaccine against human papillomavirus (HPV) includes the four HPV genotypes (6, 11,16 , and 18) that are targeted by the older quadrivalent HPV vaccine, plus five additional oncogenic types (31, 33, 45,52 , and 58) remain significantly associated with high grade lesions. We aimed to determine the prevalence of high-risk HPV genotypes in unvaccinated subjects and the association of these genotypes with the incidence of high-grade lesions. We also assessed which, if either, of these two HPV vaccines could have prevented these cases.

Methods: This cross-sectional study, conducted from 4 January 2010 to 30 December 2011, was composed of 595 women attending the Hospital General Universitario de Elche (Spain) gynaecology department who were positively screened for opportunistic cervical cancer by pap smears and HPV detection during a routine gynaecological health check. The pap smear results were classified using the Bethesda system. HPV genotyping was performed with the Linear Array HPV genotyping test, and viruses were classified by the International Agency for Research on Cancer assessment of HPV carcinogenicity. Odds ratios (ORs) with their 95\% confidence intervals (95\% Cl) were estimated by logistic regression, adjusting for age and immigrant status. The prevented fraction among those exposed (PFe-adjusted) was determined as a measure of impact.
\end{abstract}

Results: At least one of the additional five high-risk HPV genotypes present in the nine-valent HPV vaccine was detected in $20.5 \%$ of subjects. After excluding women with genotype 16 and/or 18 co-infection, high-risk genotypes (31, 33, 45, 52, and 58) were associated with a higher risk of intraepithelial lesion or malignancy: adjusted $\mathrm{OR}=3.51(95 \% \mathrm{Cl}, 1.29-9.56)$, PFe-adjusted $=0.72$ (95\% Cl, 0.22-0.90). Genotypes that are still non-vaccine-targeted were detected in $17.98 \%$ of the women, but these were not significantly associated with high-grade lesions.

Conclusion: The greater protection of the nine-valent HPV vaccine is likely to have a positive impact because, in the absence of genotype 16 or 18 infection, these five genotypes on their own remained significantly associated with high-grade lesions.

\footnotetext{
*Correspondence: maria.paz@unican.es

${ }^{1}$ Faculty of Nursing, University of Cantabria, Avda Valdecilla s/n. C.P.: 39008,

Cantabria, Spain

Full list of author information is available at the end of the article
} 


\section{Background}

Human papillomavirus (HPV) infection is the most common sexually transmitted infection in the United States and Europe [1]. Persistent HPV infection with a high-risk oncogenic genotype, as well as co-infection with high-risk genotypes, contributes to neoplastic progression [2-4]. Cervical cancer (CC) and other HPVrelated cancers represent a major global public health problem. Cervical cancer is the third most common cancer among women worldwide [5]. It has been found that according to the severity of cervical lesions, the overall prevalence of HPV increases from $12 \%$ in women with normal cytology to $89 \%$ in women with CC [6]. The most frequent viral types are HPV 16, 18, 31, 33, 35, 45, 52 and 58. However, there are differences of specific type prevalences according to the region analysed. HPV 16 and 18 are the two most prevalent types worldwide [7]. HPV genotypes 16 and 18 cause $70 \%$ of cervical cancer cases as well as an even higher proportion of other cancers associated with HPV infection, such as cancer of the vulva, vagina, penis, anus, and oropharynx [8-10].

Two preventive strategies are used in combination to prevent cervical cancer: vaccination against HPV (primary prevention) and cervical screening programmes (secondary prevention). Cervical smears allow the detection of pre-cancerous lesions and cancer. Although it is a very effective method, its implementation only helps to prevent cervical cancer $[11,12]$; there are no screening tests for other types of HPV-related cancer. In Spain, cervical screening is available but not mandatory. Cervical screening is performed opportunistically, its success depends on participant uptake.

In Europa, there are currently three vaccines authorised against HPV. The bivalent HPV vaccine Cervarix $^{\bullet}$ provides protection against HPV 16 and 18 (high-risk oncogenic genotypes); the quadrivalent HPV vaccine Gardasil $^{\odot}$ provides protection against HPV 6 and 11 (low-risk oncogenic genotypes related with the appearance of $90 \%$ of genital warts) [13] together with genotypes 16 and 18. Nowadays in Spain, we use bivalent HPV vaccine Cervarix ${ }^{\bullet}$ and quadrivalent HPV vaccine Gardasil ${ }^{\circ}$. Depending on the region, one or other vaccine is included in the childhood immunization schedule. The last approved nine-valent HPV vaccine Gardasil9 ${ }^{\circ}$ provides protection against $\operatorname{HPV} 6,11,16,18$, $31,33,45,52$, and 58 [14]. This new vaccine extends protection to a further five oncogenic types (HPV 31, 33, 45,52 , and 58), in addition to the four types included in the original Gardasil ${ }^{\circ}$. Thus, the nine-valent HPV vaccine is estimated to potentially prevent close to $90 \%$ of cancers of the cervix, vulva, vagina, and anus, as well as $80 \%$ of pre-cancerous lesions [15]. Scientific evidence regarding the safety profile [16], immunogenicity [17, 18], and safety and efficacy [19-22] of these vaccines is well established.
The aims of our study were to determine the prevalence of high-risk HPV genotypes and analyse how effective the protection offered by the quadrivalent HPV vaccine and the new nine-valent HPV vaccine would be in this population as well as to investigate the association with and potential impact of these genotypes on the incidence of high-grade lesions.

\section{Methods}

\section{Type of study}

This was a cross-sectional study of 595 women.

\section{Population}

During the study period (January 4,2010 to December 30,2011 ), women who came to the gynecology department of the Hospital General Universitario de Elche (Spain) for a routine gynecological check-up were treated according to the cervical cancer screening protocol: a combined Pap smear test and HPV screening. For our study, we included 595 consecutive women in whom the linear combined HPV genotyping test had detected HPV infection in a cervical smear.

\section{Data sources}

We obtained the data for each patient from computerized hospital records of gynaecology and microbiology services. When necessary, this information was completed with data from paper medical records and the histopathology department Cliniviewer.

\section{Variables}

The primary variables included date of birth, country of birth, patient's VPH vaccine status, Pap smear result, and determination of HPV genotype by polymerase chain reaction (PCR) assay.

The results of the pap smears were classified according to the Bethesda 2001 histological classification [23]. Positive pap smears were defined as those that presented abnormal epithelial cells. The result was classified as: atypical squamous cells of undetermined significance or atypical glandular cells (ASC-US/AGC), low grade squamous intraepithelial lesion (LSIL), high grade squamous intraepithelial lesion (HSIL), squamous cell carcinoma, adenocarcinoma in situ, or adenocarcinoma.

HPV genotyping was performed using the Linear Array HPV genotyping test (Roche Diagnostics), a qualitative in vitro assay that utilizes the amplification of target DNA by PCR and nucleic acid hybridization and detects 37 HPV genotypes: 6, 11, 16, 18, 26, 31, 33, 35, $39,40,42,45,51,52,53,54,55,56,58,59,61,62,64$, 66, 67, 68, 69, 70, 71, 72, 73 (MM9), 81, 82 (MM4), 83 (MM7), 84 (MM8), IS39, and CP6108. HPV types were classified according to the World Health Organization (WHO) International Agency for Research on Cancer (IARC) Monographs Working Group assessment of the 
carcinogenicity of different HPV types [24-26]: 13 highrisk $(16,18,31,33,35,39,45,51,52,56,58,59$, and 68$)$ and 5 likely high-risk (53, 66, 70, 73 MM9, and 82 MM4). The finding of two or more high-risk genotypes in the same patient was defined as co-infection [27].

Based on the protection that would have been provided by the quadrivalent or new nine-valent HPV vaccines, the high-risk genotypes were classified into [28]:

a) vaccine-targeted high-risk HPV genotypes (16 and 18);

b) quadrivalent HPV-targeted genotypes $(6,11,16$, and 18);

c) the additional five high-risk HPV genotypes present in the nine-valent HPV vaccine (31, 33, 45, 52, and 58);

d) high-risk HPV types other than 16 and 18 (31, 33, $35,39,45,51,52,56,58,59$, and 68); and

e) other non-vaccine targeted high-risk types (35, 39, $51,56,59$, and 68 ).

\section{Statistical analysis}

For the categorical and discrete variables, proportions were estimated using the Pearson Chi-squared test to make comparisons and using Fisher's exact test when necessary. Continuous variables were expressed as the mean and standard deviation (SD); a Student's $t$-test was used for comparisons following normality testing using a Shapiro-Wilk test.

Cytological results were classified as being a "Non-highgrade lesion" (smear negative for intraepithelial lesion,
ASC-US/AGC, or LSIL) or a "High-grade lesion" (HSIL, squamous cell carcinoma, adenocarcinoma in situ, or adenocarcinoma), and this classification was treated as a dependent variable in the logistic regression models.

As a measure of association, the crude odds ratios (ORc) as well as the odds ratios adjusted (ORa) for age (continuous variable) and immigrant status were estimated by unconditional logistic regression, together with their $95 \%$ confidence intervals $(95 \% \mathrm{CI})$.

As a measure of impact, the adjusted attributable fraction in the total exposed (AFe-adjusted) was calculated using the formula [(ORa - 1)/ORa] together with its $95 \%$ CI using the formula [((lower limit $95 \% \mathrm{CI}$ of the ORa 1)/lower limit $95 \% \mathrm{CI}$ of the ORa) to ((upper limit 95\%CI of the ORa -1)/upper limit 95\% CI of the ORa)] when the ORa was statistically significant. The alpha error was set at 0.05 , and all $p$ were two-sided. All statistical analyses were performed using IBM SPSS Statistics V22.0.

\section{Results}

Of the total participants, 73.11\% (95\% CI, 69.43-76.76) of women presented with at least one high-risk HPV genotype. High-risk HPV genotypes not targeted by the quadrivalent HPV vaccine were found in $38.49 \%$ of women $(95 \%$ CI, 34.49-42.48\%). At least one of the additional five high-risk HPV genotypes present in the nine-valent HPV vaccine (31, $33,45,52$, or 58 ) was found in $20.50 \%$ of women $(95 \%$ CI, 17.18-23.83\%) (Table 1).

Table 1 High-risk HPV genotypes, vaccine-targeted high-risk HPV genotypes, and single type infections and multiple type infections

\begin{tabular}{|c|c|c|c|c|c|c|c|c|c|c|c|c|}
\hline & Total & & & & Single HPV type & & & & Multiple HPV types ${ }^{a}$ & & & \\
\hline & $n=595$ & $\%$ & $95 \% \mathrm{Cl}$ & & $n=595$ & $\%$ & $95 \% \mathrm{Cl}$ & & $n=595$ & $\%$ & $95 \% \mathrm{Cl}$ & \\
\hline \multicolumn{13}{|c|}{ HPV genotypes } \\
\hline \multicolumn{13}{|c|}{ Vaccine-targeted high-risk HPV genotypes } \\
\hline 16 & 180 & 30.08 & 26.32 & 33.85 & 65 & 10.92 & 8.33 & 13.52 & 115 & 19.33 & 16.07 & 22.59 \\
\hline 18 & 32 & 5.38 & 3.48 & 7.28 & 6 & 1.01 & 0.12 & 1.90 & 26 & 4.37 & 2.64 & 6.10 \\
\hline \multicolumn{13}{|c|}{ Additional five high-risk HPV genotypes present in the nine-valent HPV vaccine } \\
\hline 31 & 56 & 9.41 & 6.98 & 11.84 & 19 & 3.19 & 1.70 & 4.69 & 37 & 6.22 & 4.19 & 8.24 \\
\hline 33 & 27 & 4.71 & 2.92 & 6.49 & 6 & 1.01 & 0.12 & 1.90 & 21 & 3.53 & 1.96 & 5.10 \\
\hline 45 & 18 & 3.03 & 1.57 & 4.49 & 4 & 0.67 & 0.18 & 1.71 & 14 & 2.35 & 1.05 & 3.66 \\
\hline 52 & 85 & 14.29 & 11.39 & 17.18 & 8 & 1.35 & 0.34 & 2.35 & 77 & 12.94 & 10.16 & 15.72 \\
\hline 58 & 37 & 6.22 & 4.19 & 8.24 & 4 & 0.67 & 0.18 & 1.71 & 33 & 5.55 & 3.62 & 7.47 \\
\hline \multicolumn{13}{|c|}{ Quadrivalent HPV and nine-valent HPV non-vaccine-targeted high-risk HPV genotypes } \\
\hline 35 & 17 & 2.86 & 1.43 & 4.28 & 3 & 0.50 & 0.10 & 1.47 & 14 & 2.35 & 1.05 & 3.66 \\
\hline 39 & 46 & 7.73 & 5.50 & 9.96 & 9 & 1.51 & 0.45 & 2.58 & 37 & 6.22 & 4.19 & 8.24 \\
\hline 51 & 69 & 11.60 & 8.94 & 14.25 & 14 & 2.35 & 1.05 & 3.66 & 55 & 9.24 & 6.83 & 11.66 \\
\hline 56 & 55 & 9.24 & 6.83 & 11.66 & 2 & 0.34 & 0.04 & 1.21 & 53 & 8.91 & 6.54 & 11.28 \\
\hline 59 & 28 & 4.71 & 2.92 & 6.49 & 6 & 1.01 & 0.12 & 1.90 & 22 & 3.70 & 2.10 & 5.30 \\
\hline 68 & 13 & 2.19 & 0.93 & 3.44 & 0 & 0.00 & 0.00 & 0.62 & 13 & 2.19 & 0.93 & 3.44 \\
\hline
\end{tabular}

${ }^{a}$ Women infected with multiple human papillomavirus (HPV) types: high-risk, likely high-risk HPV or low-risk genotypes [11] 
High-risk HPV genotypes not targeted by either the quadrivalent $\mathrm{HPV}$ or the nine-valent $\mathrm{HPV}$ vaccine (35, $39,51,56,59$, or 68 ) were found in $17.98 \%$ of women (95\% CI, 14.81-21.15\%) (Table 1).

The mean age of the women included in this study was 34.34 years [SD: 10.70]; 89.75\% were Spanish. Of the participants, $41.36 \%$ had at least one smear with morphological changes (ASC-US, LSIL, HSIL, squamous cell carcinoma, adenocarcinoma in situ, or adenocarcinoma). The prevalence of ASC-US was $8.14 \%$ (95\% CI, 5.8510.43), that of LSIL was $19.49 \%$ (95\% CI, 16.21-22.77), and that of HSIL was $11.53 \%$ (95\% CI, 8.86-14.19). The prevalence of cancerous lesions (squamous cell carcinoma, adenocarcinoma in situ, or adenocarcinoma) was 2.20\% (95\% CI, 0.93-3.47) (Additional file 1).

A single low-risk genotype $(6,11,42,43$ or 44$)$ was detected in $33.61 \%$ of the women (95\% CI, 29.73-37.49), and a single likely high-risk genotype was detected in 25.88\% (95\% CI, 22.28-29.49). A single high-risk genotype was detected in $45.04 \%$ of participants (95\% CI, 40.96-49.12), and two high-risk genotypes were detected in the $20.50 \%$ (Additional file 1). The most common multiple infections were by genotype 16 (19.33\%), 52 $(12.94 \%)$, or $51(9.24 \%)$ (Table 1$)$. Genotype 16 was the most commonly detected HPV genotype, found in $30.08 \%$ of study participants (95\% CI, 26.32-33.85). Genotypes 16 and/or 18 were detected in $35.46 \%$ of the women (Table 2). When we analysed the percentages of infection prevalence for the additional five high-risk HPV genotypes present in the nine-valent HPV vaccine, we found that $14.29 \%$ of women were infected by genotype $52,9.41 \%$ by genotype $31,6.22 \%$ by genotype 58 , $4.71 \%$ by genotype 33 , and $3.03 \%$ by genotype 45 (Table 1 ). Regarding the percentages of infection by high-risk HPV genotypes that are not targeted by either the quadrivalent or nine-valent HPV vaccine, we found that $11.60 \%$ of subjects were infected by genotype $51,9.24 \%$ by genotype $56,7.73 \%$ by genotype $39,4.71 \%$ by genotype $59,2.86 \%$ by genotype 35 , and $2.19 \%$ by genotype 68 (Table 2).

Table 3 shows the crude and adjusted measures of association (OR) and impact (AFe) of the risk of high-grade lesions, in relation to the HPV genotype and its potential protection by the quadrivalent and nine-valent HPV vaccines. After adjusting for age and immigrant status, the presence of HPV genotype 16 or 18 individually increased the possibility of having a high-grade lesion by 10.40 -fold. The concomitant presence of both of these genotypes increased the risk of lesion as follows: adjusted $\mathrm{OR}=63.58$; linear $p$ trend <0.001; $\mathrm{AFe}=0.98$ (95\% CI, 0.88-1.00). The presence of a high-risk genotype not covered by the quadrivalent HPV vaccine increased the risk of a high-grade lesion significantly, although to a lesser extent than the presence of genotype 16 or 18 : adjusted OR $=2.71$ (95\% CI, 1.05-7.01).
Table 2 Characteristics of the studied population, distribution of HPV genotypes, and histological classification of cervical smears

\begin{tabular}{|c|c|c|c|c|}
\hline \multirow{3}{*}{ Age (years): Mean [SD] } & \multicolumn{4}{|l|}{ Total } \\
\hline & \multirow{2}{*}{$\begin{array}{l}n=595 \\
34.34\end{array}$} & \multirow{2}{*}{$\frac{\%}{[10.70]}$} & \multicolumn{2}{|l|}{$95 \% \mathrm{Cl}$} \\
\hline & & & 33.48 & 35.20 \\
\hline \multicolumn{5}{|l|}{ Country of origin } \\
\hline Spain & 534 & 89.75 & 87.23 & 92.27 \\
\hline Other & 61 & 10.25 & 7.73 & 12.77 \\
\hline \multicolumn{5}{|l|}{ Number low-risk oncogenic HPV ${ }^{a}$} \\
\hline 1 genotype & 200 & 33.61 & 29.73 & 37.49 \\
\hline 2 genotypes & 63 & 10.59 & 8.03 & 13.15 \\
\hline 3 genotypes & 15 & 2.52 & 1.18 & 3.87 \\
\hline 4 genotypes & 6 & 1.01 & 0.12 & 1.90 \\
\hline \multicolumn{5}{|c|}{ Number likely high-risk oncogenic HPV ${ }^{a}$} \\
\hline 1 genotype & 154 & 25.88 & 22.28 & 29.49 \\
\hline 2 genotypes & 12 & 2.02 & 0.80 & 3.23 \\
\hline 3 genotypes & 1 & 0.17 & 0.00 & 0.93 \\
\hline \multicolumn{5}{|l|}{ Number high-risk oncogenic HPV ${ }^{\mathrm{a}}$} \\
\hline 1 genotype & 268 & 45.04 & 40.96 & 49.12 \\
\hline 2 genotypes & 122 & 20.50 & 17.18 & 23.83 \\
\hline 3 genotypes & 30 & 5.04 & 3.20 & 6.88 \\
\hline 4 genotypes & 14 & 2.35 & 1.05 & 3.66 \\
\hline 5 genotypes & 1 & 0.17 & 0.00 & 0.93 \\
\hline \multicolumn{5}{|l|}{ Results cervical smear ${ }^{\mathrm{b}}$} \\
\hline NILM $^{c}$ & 272 & 46.10 & 42.00 & 50.21 \\
\hline Inflammatory & 70 & 11.86 & 9.17 & 14.56 \\
\hline Warts & 1 & 0.17 & 0.00 & 0.94 \\
\hline ASC-US/ASC-H & 48 & 8.14 & 5.85 & 10.43 \\
\hline LSIL & 115 & 19.49 & 16.21 & 22.77 \\
\hline $\mathrm{HSIL}$ & 68 & 11.53 & 8.86 & 14.19 \\
\hline Carcinoma/Adenocarcinoma & 13 & 2.20 & 0.93 & 3.47 \\
\hline Result not available & 3 & 0.51 & 0.11 & 1.48 \\
\hline Missing & 5 & & & \\
\hline
\end{tabular}

${ }^{a}$ Classification of human papillomavirus (HPV) types according to the World Health Organization International Agency for Research on Cancer (IARC) Monographs Working Group assessment of the carcinogenicity of different HPV types [25-27]

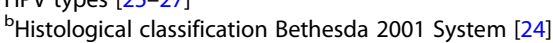

${ }^{\mathrm{c}}$ Negative for intraepithelial lesion or malignancy

By sub-dividing these genotypes based on the ability of the new nine-valent HPV vaccine to protect against them, we found that although the presence of highrisk genotypes not covered by the new nine-valent HPV vaccine was not associated with a significant increase in the risk of lesions (adjusted $\mathrm{OR}=1.76$; 95\% CI, 0.54-1.70), the presence of a high-risk genotype not covered by the quadrivalent HPV vaccine but covered by the new nine-valent HPV vaccine was associated with a significant increase in this risk 
Table 3 Prevalence of high-risk human papillomavirus genotypes according to the vaccine-targeted high-risk human papillomavirus genotypes

\begin{tabular}{|c|c|c|c|c|c|}
\hline \multirow{3}{*}{$\overline{\text { Regarding HPV genotype }}$} & & \multicolumn{4}{|l|}{ Total } \\
\hline & & \multirow[t]{2}{*}{$n=595$} & \multirow[t]{2}{*}{$\%$} & \multicolumn{2}{|l|}{$95 \% \mathrm{Cl}$} \\
\hline & & & & & \\
\hline \multicolumn{2}{|l|}{ No high-risk ${ }^{a}$} & 160 & 26.89 & 23.24 & 30.54 \\
\hline \multicolumn{2}{|l|}{ At least 1 high-risk } & 435 & 73.11 & 69.46 & 76.76 \\
\hline \multicolumn{6}{|c|}{ According to the vaccine-targeted high-risk HPV genotypes } \\
\hline \multicolumn{2}{|l|}{ No high-risk ${ }^{a}$} & 160 & 26.89 & 23.24 & 30.54 \\
\hline \multirow{3}{*}{$\begin{array}{l}\text { Quadrivalent HPV non-vaccine-targeted high-risk } \\
\text { HPV genotypes }\end{array}$} & & 229 & 38.49 & 34.49 & 42.48 \\
\hline & $\begin{array}{l}\text { Quadrivalent HPV and nine-valent HPV non-vaccine-targeted } \\
\text { high-risk HPV genotypes }^{c}\end{array}$ & 107 & 17.98 & 14.81 & 21.15 \\
\hline & $\begin{array}{l}\text { Additional five high-risk HPV genotypes present in the } \\
\text { nine-valent HPV vaccine }{ }^{d}\end{array}$ & 122 & 20.50 & 17.18 & 23.83 \\
\hline \multicolumn{2}{|l|}{$\begin{array}{l}\text { Vaccine-targeted high-risk HPV genotypes } \\
\text { (16 or } 18)\end{array}$} & 201 & 33.78 & 29.90 & 37.67 \\
\hline \multicolumn{2}{|l|}{$\begin{array}{l}\text { Vaccine-targeted high-risk HPV genotypes } \\
\text { (16 and 18) }\end{array}$} & 5 & 0.84 & 0.27 & 1.95 \\
\hline
\end{tabular}

${ }^{a}$ No high-risk. Classification human papillomavirus (HPV) types according to the World Health Organization International Agency for Research on Cancer (IARC) Monographs Working Group assessment of the carcinogenicity of different HPV types [25-27]

${ }^{\mathrm{b}}$ At least 1 of the quadrivalent HPV non-vaccine-targeted high-risk HPV genotypes (31 or 33 or 35 or 39 or 45 or 51 or 52 or 56 or 58 or 59 or 68 )

${ }^{c}$ At least 1 of the quadrivalent HPV and nine-valent HPV non-vaccine-targeted high-risk HPV genotypes (35 or 39 or 51 or 56 or 59 or 68 )

${ }^{d}$ At least 1 of the additional five high-risk HPV genotypes present in the nine-valent HPV vaccine (31 or 33 or 45 or 52 or 58 )

(adjusted OR $=3.51,95 \% \mathrm{CI}, 1.29-9.56$; adjusted AFe $=0.72,95 \%$ CI, 0.22-0.90) (Table 4).

\section{Discussion}

Our results show that in the absence of infection with HPV genotypes 16 or 18 , the greater level of protection provided by the new nine-valent HPV vaccine compared with that provided by the quadrivalent HPV vaccine is likely to have a measurable impact. The extra five HPV genotypes remained significantly associated with high grade lesions.

Our results show a high prevalence of infection by a single high-risk oncogenic HPV genotype (45.04\%) [29-32]. The percentage of women who presented a morphological change in their pap smear (positive screening) was also high $(41.36 \%)$ [33, 34]. This could be due to the characteristics of our sample, since our study was not conducted on the general population but rather it specifically included women with a confirmed HPV infection. Similarly, with respect to the prevalence of genotypes 16 and 18 in our sample, we found higher prevalences $(30.08 \%$ and $5.38 \%$, respectively) than those described in previous population-based studies [29, 32]. Our findings are closer to those reported by Garcia Espinosa et al. [35], who showed that the most common HPV genotypes found in all specimens were genotypes 16 (26.0\%), 31 (10.7\%), and 58 (8.0\%). In their study, genotype 18 was detected in only $5.0 \%$ of women.
Here, the prevalence of genotype 52 was higher than that found by other authors in national studies [36, 37]. Our results also do not coincide with the prevalence reported by the 2016 ICO-WHO report [38], which detected genotype 52 in $5.4 \%$ of HSIL and in $2.4 \%$ of cancerous lesions. This difference could be due to the high population of immigrants from East Africa that plays an important role in our health area; a very high prevalence of HPV 52 infection has been reported for this geographic area [39]. In contrast, the data published by Castellsagué et al. and Delgado et al. $[30,40]$ regarding the prevalence of HPV 52 are similar to those obtained in our study. Castellsague et al. found that after HPV 16, genotype 52 was the most prevalent, and Delgado et al. reported the prevalence for genotype 52 as $12 \%$.

The most common high-risk type detected in this study was HPV16 which is consistent with published studies and that vaccination will likely have a significant impact [20-22, 41]. Furthermore, HPV genotype 16 or 18 cause $70 \%$ of cases of cervical cancer and an even higher proportion of cancers of the vulva, vagina, penis, anus, and oropharynx [8-10]. Thus, current HPV vaccines $\left(\right.$ Cervarix $^{\circ}$ and Gardasil $^{\circ}$ ) prevent approximately $70 \%$ of cervical cancers through protection against genotypes 16 and 18 .

Partial cross-protection against non-vaccine targeted HPV types has been reported for both licensed vaccines, although the clinical significance of this finding remains uncertain [42]. In the FUTURE I / II and PATRICIA studies, conducted in young women, the bivalent vaccine 
Table 4 Associations and impact between the risk intraepithelial lesion/malignancy and the vaccine-targeted high-risk HPV genotypes

\begin{tabular}{|c|c|c|c|c|c|c|c|c|c|c|c|c|}
\hline & & No lesion & $\begin{array}{l}\text { Intraepithelial } \\
\text { lesion or malignancy }\end{array}$ & & & & & & & & & \\
\hline & & $n=509$ & $n=81$ & ORc & $95 \% \mathrm{Cl}$ & & ORa & $95 \% \mathrm{Cl}$ & & $\begin{array}{l}\text { AFe- } \\
a\end{array}$ & $95 \% \mathrm{Cl}$ & \\
\hline \multicolumn{13}{|l|}{ Regarding HPV genotype } \\
\hline No high-risk ${ }^{b}$ & & 154 & 6 & 1 & - & & 1 & - & & & & \\
\hline At least 1 high-risk & & 355 & 75 & 5.42 & 2.31 & 12.72 & 6.01 & 2.53 & 14.29 & 0.83 & 0.60 & 0.93 \\
\hline \multicolumn{13}{|c|}{ According to the vaccine-targeted high-risk HPV genotypes } \\
\hline No high-risk ${ }^{b}$ & & 154 & 6 & 1 & - & & 1 & - & & & & \\
\hline \multirow{3}{*}{$\begin{array}{l}\text { Quadrivalent HPV } \\
\text { non-vaccine-targeted } \\
\text { high-risk HPV genotypes }\end{array}$} & & 208 & 20 & 2.47 & 0.97 & 6.29 & 2.71 & 1.05 & 7.01 & 0.63 & 0.04 & 0.86 \\
\hline & $\begin{array}{l}\text { Quadrivalent HPV } \\
\text { and nine-valent } \\
\text { HPV non-vaccine- } \\
\text { targeted high-risk } \\
\text { HPV genotypes }\end{array}$ & 101 & 6 & 1.53 & 0.48 & 4.86 & 1.76 & 0.54 & 5.70 & 0.43 & - & - \\
\hline & $\begin{array}{l}\text { Additional five } \\
\text { high-risk HPV } \\
\text { genotypes present } \\
\text { in the nine-valent } \\
\text { HPV vaccine }\end{array}$ & 107 & 14 & 3.36 & 1.25 & 9.02 & 3.51 & 1.29 & 9.56 & 0.72 & 0.22 & 0.90 \\
\hline $\begin{array}{l}\text { Vaccine-targeted } \\
\text { high-risk HPV } \\
\text { genotypes ( } 16 \text { or 18) }\end{array}$ & & 145 & 52 & 9.21 & 3.84 & 22.08 & 10.40 & 4.25 & 25.40 & 0.90 & 0.76 & 0.96 \\
\hline $\begin{array}{l}\text { Vaccine-targeted } \\
\text { high-risk HPV }\end{array}$ & & 2 & 3 & 38.50 & 5.39 & 275.06 & 63.58 & 8.62 & 468.70 & 0.98 & 0.88 & 1.00 \\
\hline
\end{tabular}

$$
<0.001 \quad<0.001
$$

Intraepithelial lesion or malignancy: high-grade squamous intraepithelial lesion or worse

Odds ratio and 95\% confidence intervals. ORc denotes "crude OR"; ORa denotes "adjusted OR" OR adding to the basic model: age and immigrant status

AFe-a: Attributable fraction among the exposed, adjusted for age and immigrant status

${ }^{\mathrm{b}}$ No high-risk. Classification of human papillomavirus (HPV) types according to the World Health Organization International Agency for Research on Cancer (IARC)

Monographs Working Group assessment of the carcinogenicity of different HPV types [25-27]

${ }^{C}$ At least 1 of the quadrivalent HPV non-vaccine-targeted high-risk HPV genotypes (31 or 33 or 35 or 39 or 45 or 51 or 52 or 56 or 58 or 59 or 68 )

${ }^{\mathrm{d}}$ At least 1 of the quadrivalent HPV and nine-valent HPV non-vaccine-targeted high-risk HPV genotypes (35 or 39 or 51 or 56 or 59 or 68 )

${ }^{\mathrm{e}}$ At least 1 of the additional five high-risk HPV genotypes present in the nine-valent HPV vaccine ( 31 or 33 or 45 or 52 or 58 )

showed significant efficacy against HPV31, 33, 45 and 52, and the tetravalent vaccine against HPV31. The bivalent vaccine showed significant efficacy against CIN2 + related to HPV31 and 33 (excluding co-infection with HPV16 and / or 18) and related to HPV45 (included co-infection with HPV16 and / or 18) [43, 44]. The possible cross-protection of the new nine-valence vaccine is still unknown. It would be necessary to evaluate whether the nine-valence vaccine also protects against other non-HPV oncogenic HPV types not included in this vaccine.

Concomitant infection by both genotypes 16 and 18 also suggests an interaction, with co-infection multiplicatively increasing the risk of high-grade lesions (adjusted OR $=63.58$ ), with an adjusted AFe of $90 \%$ and $98 \%$ for isolated or joint infection, respectively. Thus, protection against infection with one of these genotypes (16 or 18) would prevent approximately $90 \%$ of high- grade lesions, while protection against both would prevent $98 \%$ of the high-grade lesions present in women co-infected with genotypes 16 and 18. These findings support the preventive impact of both the quadrivalent and the nine-valent HPV vaccine, as both cover genotypes 16 and 18 [19, 42, 45, 46].

The new nine-valent HPV virus-like particle includes the four HPV types $(6,11,16$ and 18) present in the quadrivalent HPV vaccine, plus five additional oncogenic types $(31,33,45,52$, and 58$)$ and potentially increases the overall prevention of cervical cancer from around $70 \%$ to around $90 \%$ [47]. Our study supports this increased efficacy. The greater level of protection by the new nine-valent HPV vaccine compared with that of the quadrivalent HPV vaccine is likely to have an observable impact, given our finding that these genotypes on their own (in the absence of infection by genotypes 16 or 18) remained significantly associated with high grade 
lesions, with an adjusted AFe of $72 \%$. Thus, our results suggest that had the 121 patients who presented with at least one of these HPV genotypes (but not genotype 16 or 18 ) been vaccinated with the new nine-valent HPV vaccine, approximately $72 \%$ of the high-grade lesions present in these women would have been prevented. In other words, if all these 121 patients had been vaccinated, and the efficacy of the vaccination for these genotypes (31, 33, 45, 52, and 58) was $100 \%$, approximately 87 high-grade lesions would have been prevented. Joura et al. showed an efficacy of $96.7 \%$ against high-grade lesions related with infection by HPV 31, 33, 45, 52, and 58 in their analysis of a cohort of 14,215 women aged 16 to 26 years [22]. Monsonego et al. showed that a high proportion of high-grade cervical lesions $(60.6 \%$ of genotyping assay-positive $\mathrm{CIN} 2+$ ) were associated with HPV types $31,33,45,52$, or 58 [48].

In our study, $17.98 \%$ of women were infected with high-risk genotypes that are not covered by either the quadrivalent or the new nine-valent HPV vaccine. In the absence of infection with genotypes 16 or 18 , infection with the remaining high-risk genotypes not covered by the new nine-valent HPV vaccine (genotypes $35,39,51,56,59$, and 68) was associated with a non-statistically significant risk of high-grade lesions, with an AFe close to $40 \%$.

When extrapolating our results, various aspects that could result in lower attributable fractions in the population must be considered. The effectiveness of the HPV vaccines is likely less than $100 \%$, and therefore the protection level is not $100 \%$ in $100 \%$ of the women vaccinated. Furthermore, we cannot rule out the false sense of security reported by several authors [49, 50], which can lead to a higher prevalence of risky lifestyles or sexual habits, such as a lower use of condoms. Another limitation is the fact that the attribution of lesions to specific HPV types is complicated by the detection of multiple HPV infections. Therefore, the potential benefit of a HPV vaccine is not easily assessable.

On the other hand, another caveat is that cervical lesion was performed on exfoliated cervical cells instead of biopsy samples with laser dissection, so we did not have confirmation of high-grade lesions by histological study (biopsy). However, studies evaluating the category HSIL compared to biopsy as gold standard support a very high probability of an accurate diagnosis $[47,51]$.

In our study, the enrolled women had attended the gynaecology department of the Hospital General Universitario de Elche (eastern Spain) testing positive for HPV infection in the cervical smear performed during a routine gynaecological health check in an opportunistic cervical screening program. Since the population is limited to those attending opportunistic screening further selected on the basis of their HPV status and not primarily vaccine status, it prevents to make generalizations on potential vaccine impact in the general population. However, our results circumscribed to the study population, show the prevalence of high-risk HPV genotypes in unvaccinated subjects and the association of these genotypes with the presence of high-grade lesions, allowing to assess the potential impact of quadrivalent and ninevalent HPV vaccines by using epidemiological impact measures. One advantage of using adjusted ORs is that derived from these adjusted OR, it is possible to estimate as a measure of impact, the adjusted attributable fraction in the total exposed together with its 95\%CI. We think that our methodology can complement the information provided with other methodologies such as the one followed by the recently published study by Capra et al. [52], and that it could be useful for future reviews or meta-analysis. The description of high-risk HPV epidemiology is a key feature for the design of strategies to prevent cervical cancer, and the results from this study can be used with this purpose in future reviews and meta-analysis, in order to estimate the potential impact of vaccination by either the quadrivalent or nine-valent vaccine relative to other studies with similar aims.

\section{Conclusion}

HPV genotypes 16 and 18, especially in co-infection, are the two HPV genotypes that have most impact on the presence of high-grade lesions in our unvaccinated population attending an opportunistic screening in eastern Spain. Based on our associations and impact epidemiological results, in the absence of infection with HPV genotypes 16 or 18, the greater level of protection provided by the new nine-valent HPV vaccine (five genotypes) compared with that provided by the quadrivalent HPV vaccine is likely to have a measurable impact because these genotypes on their own remained significantly associated with high-grade lesions. In our population, there is a percentage of women (17.98\%) who are infected with high-risk HPV genotypes that are not covered by any of the currently available vaccines (quadrivalent HPV or nine-valent HPV vaccines); however, as a whole, the impact of these HPV genotypes on the risk of high-grade lesions appears to be lower than the risk posed by other common HPV genotypes.

\section{Additional file}

Additional file 1: Characteristics of the studied population, distribution of HPV genotypes, and histological classification of cervical smears. (DOCX $18 \mathrm{~kb})$

\section{Abbreviations}

AFe: Adjusted attributable fraction in the exposures; AGC: Atypical glandular cells; ASC-US: Atypical squamous cells of undetermined significance; 
Cl: Confidence intervals; HPV: Human papillomavirus; HSIL: High grade squamous intraepithelial lesion; IARC: World health organization international agency for research on cancer; LSIL: Low grade squamous intraepithelial lesion; OR: Odds ratios; ORa: Odds ratios adjusted; ORc:: Crude odds ratios; PCR: Polymerase chain reaction; PFE: Prevented fraction among the exposed; SD: Standard deviation

\section{Acknowledgements}

The authors would like to thank the study participants and the clinicians, nurses and laboratory technicians of University General Hospital of Elche who were involved in the study.

\section{Funding}

This work was supported by the "Fundación para la Investigación del Hospital Universitario de Elche" (FIBELX) and the private Fundación Navarro Tripodi. The funders were not involved in the study design, data collection, analysis, or manuscript writing nor in the decision to submit the manuscript for publication.

\section{Availability of data and materials}

The dataset supporting the conclusions of this article are available upon request to the corresponding author.

\section{Authors' contributions}

MPZ, MS, JCRD were responsible for the study conception and design; MPZ and LAP were responsible for data interpretation and manuscript preparation. PPB interpreted analyses and edited manuscript. MEAB and JMRI managed the study including data collection and conceived manuscript. MMRG and JP made critical revisions to the paper for important intellectual content. All authors read and approved the final version of manuscript.

\section{Ethics approval and consent to participate}

The research protocol was approved by the Elche clinical research ethics committee and data were anonymised prior to statistical analysis. Data were treated anonymously and confidentially under Spanish Organic Law 15/1999 of 13th December, on Personal Data Protection.

The written human subject consent was not considered necessary by the Elche clinical research ethics committee. Our study was a cross-sectional study and we used secondary records of gynaecology and microbiology services.

\section{Consent for publication}

\section{Not Applicable.}

\section{Competing interests}

The authors declare that they have no competing interests.

\section{Publisher's Note}

Springer Nature remains neutral with regard to jurisdictional claims in published maps and institutional affiliations.

\begin{abstract}
Author details
${ }^{1}$ Faculty of Nursing, University of Cantabria, Avda Valdecilla s/n. C.P.: 39008 , Cantabria, Spain. ${ }^{2}$ Department of Microbiology and Parasitology, University Hospital of Burgos, C/ Islas Baleares, 3 - C.P.: 09006, Burgos, Spain. ${ }^{3}$ Department of Microbiology, University General Hospital of Alicante. Pintor Baeza, 11- C.P.: 03010, Alicante, Spain. ${ }^{4}$ Department of Pathological Anatomy, University General Hospital of Elche. Camí de I'Almazara, 11 - C.P.: 03203, Alicante, Spain. ${ }^{5}$ Department of Gynecology, University General Hospital of Elche. Camí de I'Almazara, 11 - C.P.: 03203, Alicante, Spain. ${ }^{6}$ Department of Microbiology, University General Hospital of Elche. Camí de I'Almazara, 11 C.P.: 03203, Alicante, Spain. ${ }^{7}$ Department of Infectious Diseases, University Hospital of Alicante. Pintor Baeza, 11- C.P.: 03010, Alicante, Spain.
\end{abstract}

Received: 10 March 2017 Accepted: 23 January 2018

Published online: 30 January 2018

\section{References}

1. Centers for Disease Control and Prevention. Genital HPV infection fact sheet. Rockville: MD: CDC National Prevention Information Network; 2014.

2. Hartwig S, Baldauf JJ, Dominiak-Felden G, Simondon F, Alemany L, de Sanjosé S, et al. Estimation of the epidemiological burden of HPV-related anogenital cancers, precancerous lesions, and genital warts in women and men in Europe: potential additional benefit of a nine-valent second generation HPV vaccine compared to first generation HPV vaccines. Papillomavirus Res. 2015;1:90-100.

3. Carrillo-García A, Ponce-de-León-Rosales S, Cantú-de-León D, FragosoOntiveros V, Martínez-Ramírez I, Orozco-Colín A, et al. Impact of human papillomavirus coinfections on the risk of high-grade squamous intraepithelial lesion and cervical cancer. Gynecol Oncol. 2014;134(3):534-9.

4. Chelimo C, Wouldes TA, Cameron LD, Elwood JM. Risk factors for and prevention of human papillomaviruses (HPV), genital warts and cervical cancer. J Inf Secur. 2013;66(3):207-17.

5. Ferlay J, Soerjomataram I, Dikshit R, Eser S, Mathers C, Rebelo M, et al. Cancer incidence and mortality worldwide: sources, methods and major patterns in GLOBOCAN 2012. Int J Cancer. 2014;136(5):E359-86.

6. Guan P, Howell-Jones R, Li N, Bruni L, de Sanjose S, Franceschi S, et al. Human papillomavirus types in 115,789 HPV-positive women: a metaanalysis from cervical infection to cancer. Int J Cancer. 2012;131(10):2349-59.

7. Li N, Franceschi S, Howell-Jones R, Snijders PJ, Clifford GM. Human papillomavirus type distribution in 30,848 invasive cervical cancers worldwide: variation by geographical region, histological type and year of publication. Int J Cancer. 2010;128(4):927-35.

8. De Sanjose S, Quint WG, Alemany L, Geraets DT, Klaustermeier JE, Lloveras $B$, et al. Human papillomavirus genotype attribution in invasive cervical cancer: a retrospective cross-sectional worldwide study. Lancet Oncol. 2010; 11:1048-56.

9. Gillison ML, Chaturvedi AK, Lowy DR. HPV prophylactic vaccines and the potential prevention of noncervical cancers in both men and women. Cancer. 2008;113:3036-46

10. Forman D, de Martel C, Lacey CJ, Soerjomataram I, Lortet-Tieulent J, Bruni L, et al. Global burden of human papillomavirus and related diseases. Vaccine. 2012;30(Suppl 5):F12-23.

11. Arbyn M, Anttila A, Jordan J, Ronco G, Schenck U, Segnan N, et al. European guidelines for quality assurance in cervical cancer screening. 2nd ed. summary document. Ann Oncol. 2010;21:448-58.

12. Saslow D, Solomon D, Lawson HW, Killackey M, Kulasingam SL, Cain J, et al. American Cancer Society, American Society for Colposcopy and Cervical Pathology, and American Society for Clinical Pathology screening guidelines for the prevention and early detection of cervical cancer. CA Cancer J Clin. 2012;62(3):147-72

13. Centers for Disease Control and Prevention. HPV vaccine information for clinicians-fact sheet. https://www.cdc.gov/std/HPV/. Accesed 13 Dec 2016.

14. Food and Drug Administration. FDA approves Gardasil 9 for prevention of certain cancers caused by five additional types of HPV. https://www.fda. gov/BiologicsBloodVaccines/Vaccines/ApprovedProducts/ucm426445.htm. Accessed 13 Dec 2016.

15. Joura EA, Ault KA, Bosch FX, Brown D, Cuzick J, Ferris D, et al. Attribution of 12 high-risk human papillomavirus genotypes to infection and cervical disease. Cancer Epidemiol Biomark Prev. 2014;23:1997-2008.

16. World Health Organization. Global advisory committee on vaccine Safety,12-13 June 2013. Wkly Epidemiol Rec. 2013;88:301-12.

17. Schwarz TF, Spaczynski M, Schneider A, Wysocki J, Galaj A, Perona P, et al. Immunogenicity and tolerability of an HPV-16/18 AS04-adjuvanted prophylactic cervical cancer vaccine in women aged 15-55 years. Vaccine. 2009;27:581-7.

18. Castellsagué X, Muñoz N, Pitisuttithum P, Ferris D, Monsonego J, Ault K, et al. End of study safety, immunogenicity, and efficacy of quadrivalent HPV (types $6,11,16,18$ ) recombinant vaccine in adult women $24-45$ years of age. Br J Cancer. 2011;105:28-37.

19. Lehtinen M, Paavonen J, Wheeler CM, Jaisamrarn U, Garland SM, Castellsagué X, et al. Overall efficacy of HPV-16/18 AS04-adjuvanted vaccine against grade 3 or greater cervical intraepithelial neoplasia: 4-year end-ofstudy analysis of the randomised, double-blind PATRICIA trial. Lancet Oncol. 2012;13:89-99.

20. Garland SM, Hernandez-Avila M, Wheeler CM, Perez G, Harper DM, Leodolter $S$, et al. Quadrivalent vaccine against human papillomavirus to prevent anogenital diseases. N Engl J Med. 2007;356:1928-43.

21. Future II Study Group. Quadrivalent vaccine against human papillomavirus to prevent high-grade cervical lesions. N Engl J Med. 2007;356:1915-27.

22. Joura EA, Giuliano AR, Iversen OE, Bouchard C, Mao C, Mehlsen J, et al. 9valent HPV vaccine against infection and intraepithelial neoplasia in women. N Engl J Med. 2015;372(8):711-23. 
23. Solomon D, Davey D, Kurman R, Moriarty A, O'Connor D, Prey M, et al. Forum group members; Bethesda 2001 workshop. The 2001 Bethesda system. Terminology for reporting results of cervical cytology. JAMA. 2002;287:2114-9.

24. Muñoz N, Bosch FX, de Sanjose S, Herrero R, Castellsague X, Shah KV, et al. Epidemiologic classification of human papillomavirus types associated with cervical cancer. N Engl J Med. 2003;348(6):518-27.

25. IARC. Human papillomaviruses. IARC Monogr Eval Carcinog Risks Hum. 2007;90:1-636.

26. Schiffman M, Clifford G, Buonaguro FM. Classification of weakly carcinogenic human papillomavirus types: addressing the limits of epidemiology at the borderline. Infect Agent Cancer. 2009;4:8.

27. Trigo-Daporta M, García-Campello M, Pérez-Ríos M, Santiago-Pérez MI Fernandez-Rodriguez E, Guinarte G, et al. High-risk human papillomavirus in Galicia, Spain: prevalence and evaluation of the sample representativeness. Scand J Infect Dis. 2014;46(11):737-44.

28. Chow EP, Machalek DA, Tabrizi SN, Danielewski JA, Fehler G, Bradshaw CS, et al. Quadrivalent vaccine-targeted human papillomavirus genotypes in heterosexual men after the Australian female human papillomavirus vaccination programme: a retrospective observational study. Lancet Infect Dis. 2016; https://doi.org/10.1016/S1473-3099(16)30116-5.

29. Wright TC Jr, Stoler MH, Behrens CM, Apple R, Derion T, Wright TL. The ATHENA human papillomavirus study: design, methods, and baseline results. Am J Obstet Gynecol. 2012;206(1):46.e1-46.e11.

30. Castellsagué X, Iftner T, Roura E, Vidart JA, Kjaer SK, Bosch FX, Muñoz N, Palacios S, San Martin Rodriguez M, Serradell L, Torcel-Pagnon L, Cortes J. CLEOPATRE Spain study group. Prevalence and genotype distribution of human papillomavirus infection of the cervix in Spain: the CLEOPATRE study. J Med Virol. 2012;84(6):947-56.

31. Pista A, de Oliveira CF, Cunha MJ, Paixao MT, Real O. CLEOPATRE Portugal study group. Prevalence of human papillomavirus infection in women in Portugal: the CLEOPATRE Portugal study. Int J Gynecol Cancer. 2011;21(6):1150-8.

32. Dunne EF, Unger ER, Sternberg M, Mc Quillan G, Swan DC, Patel SS, et al. Prevalence of HPV infection among females in the United States. JAMA. 2007;297:813-9.

33. Dickson EL, Vogel Rl, Geller MA, Downs LS Jr. Cervical cytology and multiple type HPV infection: a study of 8182 women ages 31-65. Gynecol Oncol. 2014;133(3):405-8

34. Asiaf A, Ahmad ST, Mohammad SO, Zargar MA. Review of the current knowledge on the epidemiology, pathogenesis, and prevention of human papillomavirus infection. Eur J Cancer Prev. 2014;23:206-24.

35. García-Espinosa B, Moro-Rodríguez E, Alvarez-Fernández E. Genotype distribution of human papillomavirus (HPV) in histological sections of cervical intraepithelial neoplasia and invasive cervical carcinoma in Madrid, Spain. BMC Cancer. 2012;12:533.

36. Otero-Motta AP, Ordonez JL, Gonzalez-Celador R, Rivas B, Macias Mdel C Bullon A, et al. Prevalence of human papillomavirus genotypes in cytologic abnormalities from unvaccinated women living in north-western Spain. APMIS. 2011;119:204-15.

37. Cobo F, Concha A, Ortiz M. Human Papillomavirus (HPV) type distribution in females with abnormal cervical cytology. A correlation with histological study. Open Virol J. 2009;3:60-6.

38. ICO. Spain human Papillomavirus and related cancers. Information Centre on HPV and cancer, fact sheet 2016

39. De Sanjose S, Diaz M, Castellsague X, Clifford G, Bruni L, Munoz N, et al. Worldwide prevalence and genotype distribution of cervical human papillomavirus DNA in women with normal cytology: a meta-analysis. Lancet Infect Dis. 2007;7:453-9.

40. Delgado D, Marin JM, de Diego J, Guerra S, Gonzalez B, Barrios JL, et al. Human papillomavirus (HPV) genotype distribution in women with abnormal cervical cytology in the Basque Country, Spain. Enferm Infecc Microbiol Clin. 2012;30:230-5

41. Kjaer SK, Frederiksen K, Munk C, Iftner T. Long-term absolute risk of cervical intraepithelial neoplasia grade 3 or worse following human papillomavirus infection: role of persistence. J Natl Cancer Inst. 2010;102:1478-88.

42. Schiller JT, Castellsagué X, Garland SM. A review of clinical trials of human papillomavirus prophylactic vaccines. Vaccine. 2012;30(Suppl 5):F123-38.

43. Brown DR, Kjaer SK, Sigurdsson K, Iversen OE, Hernandez-Avila M, Wheeler $\mathrm{CM}$, et al. The impact of quadrivalent human papillomavirus (HPV; types 6, 11,16 , and 18) L1 virus-like particle vaccine on infection and disease due to oncogenic nonvaccine HPV types in generally HPV-naive women aged 1626 years. J Infect Dis. 2009;199(7):926-35.

44. Wheeler CM, Castellsagué X, Garland SM, Szarewski A, Paavonen J, Naud P, et al. Cross-protective efficacy of HPV-16/18 AS04-adjuvanted vaccine against cervical infection and precancer caused by non-vaccine oncogenic HPV types: 4-year end-of-study analysis of the randomised, double-blind PATRICIA trial. Lancet Oncol. 2012;13(1):100-10.

45. Muñoz N, Kjaer SK, Sigurdsson K, Iversen O-E, Hernandez-Avila M, Wheeler CM, et al. Impact of human papillomavirus (HPV)-6/11/16/18 vaccine on all HPV-associated genital diseases in young women. J Natl Cancer Inst. 2010; 102:325-39.

46. Hildesheim A, Wacholder S, Catteau G, Struyf F, Dubin G, Herrero R, et al. Efficacy of the HPV-16/18 vaccine: final according to protocol results from the blinded phase of the randomized Costa Rica HPV-16/18 vaccine trial. Vaccine. 2014;32:5087-97.

47. Serrano B, Alemany L, Tous S, Bruni L, Clifford GM, Weiss T, et al. Potential impact of a nine-valent vaccine in human papillomavirus related cervical disease. Infect Agent Cancer. 2012;7:38.

48. Monsonego J, Zerat L, Syrjänen K, Zerat JC, Smith JS, Halfon P. Prevalence of type-specific human papillomavirus infection among women in France: implications for screening, vaccination, and a future generation of multivalent HPV vaccines. Vaccine. 2012;30(35):5215-21.

49. Henderson L, Clements A, Damery S, Wilkinson C, Austoker J, Wilson S. HPV Core messages writing group. 'A false sense of security'? Understanding the role of the HPV vaccine on future cervical screening behaviour: a qualitative study of UK parents and girls of vaccination age. J Med Screen. 2011;18(1):41-5.

50. Hendry M, Lewis R, Clements A, Damery S, Wilkinson C, HPV? Never heard of it': a systematic review of girls' and parents' information needs, views and preferences about human papillomavirus vaccination. Vaccine. 2013;31(45):5152-67.

51. Mukhopadhyay S, Ray S, Dhar S, Bandyopadhyay R, Sinha SK. Evaluation of the category high-grade squamous intraepithelial lesion in the Bethesda system for reporting cervical cytology. J Cytol. 2013;30(1):33-5.

52. Capra G, Giovannelli L, Matranga D, Bellavia C, Guarneri MF, Fasciana T, et al. Potential impact of a nonavalent HPV vaccine on HPV related low-and highgrade cervical intraepithelial lesions: a referral hospital-based study in Sicily. Hum Vaccin Immunother. 2017;13(8):1839-43.

\section{Submit your next manuscript to BioMed Central and we will help you at every step:}

- We accept pre-submission inquiries

- Our selector tool helps you to find the most relevant journal

- We provide round the clock customer support

- Convenient online submission

- Thorough peer review

- Inclusion in PubMed and all major indexing services

- Maximum visibility for your research

Submit your manuscript at www.biomedcentral.com/submit
Biomed Central 